\title{
LA POLÍTICA SOCIAL EN CUBA: NUEVA REFORMA ECONÓMICA
}

\section{SOCIAL POLICY IN CUBA: NEW ECONOMIC REFORM}

\section{Mayra Paula Espina Prieto*}

\section{RESUMEN}

El artículo ofrece un panorama sintético de las características generales del modelo de política social y laboral puesto en práctica en la transición socialista cubana y valora sus logros (amplia inclusión social, altas tasas de ocupación, bajo desempleo), así como, sus debilidades, principalmente las vinculadas a la baja capacidad de los ingresos salariales de los trabajadores para satisfacer las necesidades básicas. Describe la actual etapa de reforma económica y su potencial esperado con el fin de superar o no, estos problemas.

PALABRAS CLAVE: POLÍTICA SOCIAL * POLÍTICA SOCIOLABORAL * DESIGUALDAD SOCIAL * REFORMA ECONÓMICA

\section{ABSTRACT}

This article shows a synthetic point of view about the general characteristics of the labour and social political model, applied in the Cuban socialist transition. It evaluates the achievements (wide social inclusion, high occupation, low unemployment), as well as, the weakness related with the above mentioned model, mainly the salary low capacities to satisfies the worker basic necessities. The current economical reform and their potential to overcome this problem are described.

KEYWORDS: SOCIAL POLICY * LABOUR POLICY * SOCIAL INEQUALITY * ECONOMIC REFORM

Agencia Suiza para la Cooperación y el Desarrollo (COSUDE) y Universidad de La Habana, Cuba. mayra.espina@cosude.org 


\section{CARACTERÍSTICAS GENERALES DE LA POLÍTICA SOCIAL CUBANA EN LA TRANSICIÓN SOCIALISTA}

Partiendo de dos elementos básicos de la definición de las políticas públicas: el enfoque (noción que alude al ámbito de la conceptualización, al normativo y de los objetos y problemas sobre los cuales dichas políticas deberían actuar) y el estilo (referido al campo de la aplicación, a los actores y técnicas de intervención sobre el cambio social), puede decirse que la política social de la experiencia socialista cubana se ha caracterizado por tener como racionalidad organizadora el enfoque integrador y el estilo universalizador, que colocan el ensanchamiento progresivo de los montos y alcances de la redistribución como parámetro rector.

En la estrategia de desarrollo social seguida por la transición socialista cubana, la equidad se ha concretado como la integración de tres principios básicos: el de igualdad absoluta (expresa la exigencia ético-jurídica de completar un espacio de derechos universales básicos inalienables y oportunidades reales para que todos los ciudadanos puedan desarrollar sus capacidades sin exclusión alguna), el de solidaridad (incluye la atención preferencial diferenciada a las desventajas $y$ necesidades especiales de individuos y grupos sociales particulares, por motivo de discapacidad, ancianidad o desventajas de naturaleza socioeconómico históricas) y el de igualdad relativa o proporcional (acepta la presencia de desigualdades legítimas, asociadas al monto, la calidad y la utilidad de aportes laborales o servicios de otro tipo, individuales y colectivos).

A ello, se añade la consideración de la equidad como propuesta de norma distributiva y redistributiva. El propósito de este tipo de abordaje es el de la aplicación de criterios de equidad histórica concretos con el fin de establecer un sistema de prioridades básicas para la política social. Tales criterios, por su naturaleza histórica y cambiante como la de toda sociedad, deben ser sistemáticamente actualizados y socialmente consensuados.

La intención es que estos elementos no sean tomados como efectos subordinados de la política económica, sino que ellos constituyan, por sí mismos, dimensiones sustantivas de una estrategia de desarrollo. En esta lógica, el alivio de la pobreza y la atención a las desventajas forman parte de una política más amplia de desarrollo social y de un manejo universalista, más que focalizado.

La política sociolaboral, especialmente en el sentido de pleno empleo, ha jugado un rol central en términos de integración social y de acceso al bienestar para las amplias mayorías. El acceso al trabajo se ha considerado dentro de las necesidades básicas a satisfacer por responsabilidad estatal. En los años 2000, la tasa de actividad económica se mantuvo por encima del $70 \%$ y la de desocupación no rebasó el 2\% (Oficina Nacional de Estadísticas, 2010: 160).

El gasto social, desde el inicio de la experiencia socialista en la década de los 60 , se caracteriza por una alta prioridad macroeconómica de la esfera social. Aún en la década de los 90 , en condiciones de crisis y de reforma económica, la proporción del gasto público social con relación al PIB se mantuvo por encima del 20\% (Oficina Nacional de Estadísticas, 2010: 131-140):

GASTO PÚBLICO SOCIAL SOBRE EL PIB

\begin{tabular}{cc}
\hline AÑOS & PORCENTAJE \\
\hline $1990-1991$ & 28,4 \\
$1994-1995$ & 31,9 \\
$1998-1999$ & 41,6 \\
$1999-2000$ & 34,3 \\
$2001-2008$ & $\geq 35$ \\
\hline
\end{tabular}

Fuente: Oficina Nacional de Estadísticas, 2010.

En términos de manejo de las dimensiones sociales del desarrollo y de la promoción de equidad en una sociedad periférica, puede decirse que la experiencia cubana muestra que la universalización de los derechos sociales de ciudadanía, a través de una amplia intervención estatal y de su regulación en todas las esferas $y$ de servicios homogenizados para toda la sociedad, es una fórmula eficiente para proveer rápidamente integración social a las más amplias mayorías, aun en condiciones de poco crecimiento económico. Un cálculo de la pobreza para 1984, se estimó en un 6,3\%, proporción relativamente baja para una sociedad de recursos limitados como la cubana. 
Sin embargo, esta fórmula presenta limitaciones que se asocian al hiperestatalismo, al exceso de centralización de las políticas, al homogenismo distributivo $y$ a la baja presencia de instrumentos afirmativos, que han tendido a reproducir en el tiempo inequidades anteriores. La situación de crisis y reforma de los años 90 configuró una tendencia al ensanchamiento de las desigualdades sociales, entre cuyos rasgos más fuertes aparecen los siguientes (Espina, 2008: 135-139):

\section{A) DIFERENCIACIÓN DE LOS INGRESOS Y DEL ACCESO AL CONSUMO}

El coeficiente Gini, calculado para finales de los 90, se elevó a 0,38, superando el 0, 24 de los 80 , lo que ilustra una tendencia a la concentración de ingresos que interrumpe la lógica desconcentradora anterior (Ferriol, 2004: 128). Importantes áreas de necesidades básicas (al menos el 50\% de los requerimientos alimentarios, el vestuario, productos de aseo, materiales para reparación y equipamiento de la vivienda) solo encuentran una parte de sus satisfactores en el mercado de precios libres, de CUC o en el negro, lo que junto a la caída de la capacidad adquisitiva del salario real de los trabajadores asociada a la crisis y no recuperada aún, junto al incremento de los precios al consumidor, han reconstituido a los ingresos y al mercado como elementos de alta fuerza diferenciadora.

\section{B) INSUFICIENCIA DE LOS INGRESOS SALARIALES PARA CUBRIR LAS NECESIDADES BÁSICAS (DETERIORO DEL TRABAJO COMO MEDIO DE SATISFACCIÓN DE DICHAS NECESIDADES)}

La crisis significó un grave deterioro del salario real y hacia finales de los años 90 , se aplicaron medidas para el incremento de las pensiones $y$ de los salarios de los trabajadores estatales en ocupaciones seleccionadas (maestros y profesores, policías, científicos, personal de salud, entre otros). Hacia el año 2005, el salario medio mensual de los trabajadores ascendió a 330 pesos, superando los 203 pesos de 1996 o su monto de 284 pesos en el 2004 (Oficina Nacional de Estadísticas, 2010: 162). El salario mínimo quedó fijado en 225 pesos, las pensiones mínimas ascendieron de 65 a
164 pesos y las prestaciones de la asistencia social pasaron de 62 a 122 pesos, en virtud de la Resolución 28 de 2006 del Ministerio de Trabajo.

Pero tales incrementos en los salarios $y$ en las pensiones se ven limitados por los altos precios de muchos productos esenciales para la satisfacción de las necesidades básicas. Para 1998, se calculó una canasta básica de alimentos que alcanzó un valor de 156 pesos per cápita, demostrándose que al menos el 23,75\% de dicha población recibía ingresos salariales por debajo de la canasta calculada (Nerey, 2000: 57).

Otro estudio realizado en 2006, en el que además de los alimentos se incluyeron en la canasta otros bienes y servicios indispensables para el desarrollo de los seres humanos (como ropa y calzado, productos de aseo personal e higiene del hogar, servicios básicos) corrobora que el monto de los ingresos salariales de la población continúa siendo deficitario para la cobertura de esta canasta o está muy cercano a ese límite (García y Anaya, 2006: 22). Este estudio muestra como el per cápita mensual para la adquisición de la canasta oscilaba entre 312,50 y 330,54 pesos, mientras que el salario mínimo no rebasaba los 225 pesos mensuales y el salario medio mensual, en ese año, se situaba en 387 pesos. Aunque la situación de los ingresos es cambiante, hasta hoy no se ha mejorado la relación entre salarios y canasta básica.

\section{C) REEMERGENCIA DE SITUACIONES DE POBREZA}

Atendiendo a la pobreza de ingresos y necesidades básicas insatisfechas, la población urbana bajo esta condición, pasó a ser el $20 \%$ de la población urbana hacia el año 2000 (Ferriol, 2004: 132), lo que indica la estructuración de mecanismos distributivos excluyentes, que a diferencia de los años 80, aparecen desde el nivel de la satisfacción de necesidades básicas.

\section{D) FORTALECIMIENTO DE BRECHAS DE EQUIDAD RELACIONADAS CON EL GÉNERO Y VINCULADAS AL TRABAJO}

Los estudios de género muestran, a pesar de los avances, la persistencia de la reproducción 
de desventajas para la mujer que se concentran en los siguientes elementos:

$\diamond \quad$ Sobrerrepresentación de mujeres en los grupos de menores ingresos y en la franja de pobreza. La preferencia por las mujeres en el patrón de constitución de la pobreza en el país, se asocia a la maternidad temprana, la jefatura de hogar femenina $y$ la condición de madre soltera, circunstancias combinadas con el abandono de estudios y la ausencia de condiciones para trabajar y generar ingresos suficientes (Espina, 2008: 194). Subrepresentación de mujeres en cargos de dirección. Disminución del peso de las mujeres a medida que se asciende en el nivel de jerarquía de la dirección; distribución asimétrica del poder en la dirección de los procesos productivos, esfera donde se advierte casi una exclusión de las mujeres en la dirección (Echevarría, 2004: 15).

$\diamond \quad$ Subrepresentación de mujeres en la actividad turística. Donde solo el 36,6\% de la fuerza de trabajo es femenina, en su mayoría concentrada en ocupaciones de menor calificación y no directivas (Álvarez, 2000: 31).

$\diamond \quad$ Subrepresentación de mujeres en el sector de propiedad no estatal. Desde los años 90 y hasta la actualidad, las mujeres no rebasan el 25\% de los trabajadores autoempleados, las microempresas, cooperativas, empresas de capital mixto (nacional estatal y extranjero), espacios económicos donde los ingresos son como promedio, superiores al sector estatal (Oficina Nacional de Estadísticas, 2010: 161).

$\diamond \quad$ Diferencias de ingresos entre mujeres $y$ hombres, a favor de estos últimos. Las mujeres del sector estatal perciben salarios que representan entre un $80 \%$ y un $85 \%$ de los salarios de los hombres, lo que se relaciona con que ellas tienen un alto peso en las categorías ocupacionales de servicios $y$ administrativas (85\% y $55 \%$, respectivamente) en las que se con- centran plazas de menor remuneración $y$ tienen menor presencia en actividades estatales caracterizadas por altos ingresos (Pérez, 2004: 55).

$\diamond \quad$ Mayor carga de horas de trabajo en el hogar (más de 34 horas como promedio semanal en labores fundamentales, mientras los hombres emplean alrededor de 12 horas y básicamente en labores de apoyo). Reproducción del patrón tradicional de los roles de género (las alternativas desplegadas por las mujeres, ante la crisis, están más asociadas al ámbito doméstico, mientras que los hombres orientan sus acciones fuera del hogar). Casi exclusividad femenina en el rol de cuidadoras de niños, enfermos y personas de la tercera edad (Departamento de Estudios sobre Familia, 2001: 76).

\section{E) BRECHA DE EQUIDAD RACIALIZADA}

A partir de los datos del censo de 2002 (ONE, 2006) puede comprobarse: la sobrerrepresentación de personas no blancas en la población desocupada, su subrepresentación en cargos de dirección, de oficinas, profesionales, científicos e intelectuales, así como, entre los trabajadores autoempleados, cuyos ingresos suelen ser superiores a los de ocupaciones equivalentes en el sector estatal $y$ entre aquellos que culminan estudios superiores; mientras que están sobrerrepresentados en empleos de la construcción y la industria.

\section{F) PATRÓN SELECTIVO DE MOVILIDAD SOCIAL}

Estudios recientes de movilidad social han mostrado que el sector de los servicios (turísticos, gastronómicos y técnicos), las actividades directivas y técnicas, la economía emergente $y$ el sector no estatal de la economía (cuentapropismo, pequeña burguesía urbana, la empresa mixta y el capital extranjero) se han configurado como los espacios receptores más importantes. El sector estatal, la industria y las ocupaciones obreras, son los principales emisores. La estrechez y selectividad de los canales de movilidad social ascendente tienden a reproducir y fortalecer brechas de equidad de género, 
raza y origen social, así como, su expresión territorialmente diferenciada. La combinación de factores como la calificación, los activos familiares, tangibles e intangibles, las remesas, las redes y conexiones sociales (que puedan proveer de información oportuna, conexiones, recomendaciones $y$ privilegios) es relevante para experimentar movilidad ascendente.

\section{EL NUEVO MOMENTO DE REFORMA EN CUBA: CAMBIOS EN LA POLÍTICA SOCIAL}

El Vi Congreso del Partido Comunista de Cuba, celebrado en abril de 2011, aprobó una plataforma de cambios, oficialmente denominada "proceso de actualización del modelo económico cubano", lo cual implica la apertura de una nueva etapa de reforma que se orienta al tránsito hacia un nuevo patrón de desarrollo, cuyos vectores básicos serían (Partido Comunista de Cuba, 2011: 4-34):

$\diamond \quad$ Socialismo multiactoral.

$\diamond \quad$ Cambio en la estructura económicoproductiva: mayor peso de la sustitución de exportaciones, tercerización.

$\diamond \quad$ Municipalización (Creación de un tributo territorial que las empresas pagarán a los Consejos de Administración Municipal, donde operan sus establecimientos, con el fin de contribuir al desarrollo de la localidad. Desarrollo de proyectos locales, especialmente en producción de alimentos; orientados al autoabastecimiento municipal bajo el principio de autosustentabilidad financiera); creación de zonas especiales de desarrollo que permitan incrementar el desarrollo local; flexibilización del modelo de gestión de la industria local; desarrollo de ofertas turísticas atractivas como parte de la iniciativa municipal de los territorios y fuente de ingresos en divisas; aseguramiento de los programas de vivienda a nivel municipal, a partir de materiales existentes en cada lugar y tecnologías disponibles.

$\diamond \quad$ Descentralización empresarial y territorial.

$\diamond \quad$ Política social universal-focalizada hacia las desventajas. Conservará su estilo universalizador $y$ sus instrumentos de gra- tuidad e igualdad en áreas que se consideran básicas (educación, salud, cultura $y$ deporte). Experimentará un tránsito hacia un mayor peso de la focalización hacia grupos vulnerables $y$ de los criterios de eficiencia en el uso de los recursos y de compatibilización con las posibilidades económicas. Configuración de un mercado laboral.

La orientación hacia una economía multiactoral supone que se diversificarán los sujetos económicos no estatales (micro y pequeñas empresas privadas, cooperativas), se reforzará la articulación y la complementación entre ellos, se ampliará el papel del mercado en la distribución de bienes y servicios, así como, en el acceso al bienestar y con esto, el rol de los ingresos personales $y$ familiares en dicho acceso.

En este sentido, es necesario considerar que la política social será también objeto de reforma $y$ aun cuando de acuerdo con los lineamientos, conserve su estilo universalizador y sus instrumentos de gratuidad e igualdad en áreas que se consideran básicas (educación, salud, cultura y deporte).

Dentro del conjunto de lineamientos y medidas que forman parte de la actual estrategia de cambio, un grupo relativamente amplio tiene vínculos directos o indirectos, con la modificación de la política laboral y con la tendencia hacia la formación de un mercado de trabajo, espacio que hasta hoy no funcionaba como tal o donde el peso del mercado en el acceso laboral era muy bajo, dada la intervención directa y centralmente reglamentada de la política estatal. En ese grupo aparecen las siguientes líneas de cambio:

\section{CAMBIOS RELACIONADOS CON LA POLÍTICA DE EMPLEO-INGRESOS}

$\diamond \quad$ Reconocimiento y estímulo a las empresas de capital mixto, cooperativas, usufructuarios, arrendadores, trabajadores por cuenta propia $y$ otras formas de gestión económica.

$\diamond \quad$ Posibilidad de creación de cooperativas de segundo grado (unión de varias cooperativas de mutuo acuerdo). 
$\diamond \quad$ Continuar propiciando la participación del capital extranjero.

$\diamond \quad$ Adoptar nuevo modelo de gestión, adecuado a la mayor presencia de formas productivas no estatales y utilización más efectiva de las relaciones monetario mercantiles en la producción agroindustrial.

$\diamond \quad$ Independizar las cooperativas de la intermediación de empresas estatales.

$\diamond \quad$ Desarrollar la actividad no estatal de alojamiento, gastronomía y otros servicios como oferta turística complementaria a la estatal.

$\diamond \quad$ Adopción de formas no estatales de gestión en las labores de mantenimiento $y$ conservación del fondo habitacional.

$\diamond \quad$ Apertura de mayores espacios para las actividades no estatales en la industria local.

$\diamond \quad$ Creación de fondos empresariales para la estimulación a los trabajadores a partir de las utilidades.

$\diamond \quad$ Ingresos de los trabajadores vinculados a resultados finales.

$\diamond \quad$ Independencia empresarial para la constitución de las plantillas de cargo.

$\diamond \quad$ Acceso a créditos personales para la compra de bienes y servicios.

$\diamond \quad$ Fortalecer el papel del salario en la sociedad.

$\diamond \quad$ Política inversionista orientada prioritariamente al empleo productivo.

$\diamond \quad$ Incrementar $y$ consolidar los ingresos por concepto de exportaciones de bienes $y$ servicios.

$\diamond \quad$ Priorización del desarrollo industrial para la exportación y el mercado interno de insumos.

$\diamond \quad$ Priorizar incrementos salariales a los puestos de trabajo que generen ingresos en divisas o produzcan ahorro de las mismas.

$\diamond \quad$ Modificar la estructura del empleo: reducir el empleo estatal y ampliar el trabajo por cuenta propia y en el sector no estatal en general.

$\diamond \quad$ Formación de fuerza de trabajo calificada en correspondencia con las demandas de la economía. $\diamond \quad$ La matrícula en las diferentes carreras deberá estar en correspondencia con las demandas del desarrollo de la economía $y$ la sociedad.

\section{CAMBIOS EN EL PRESUPUESTO Y LOS GASTOS} SOCIALES

$\diamond \quad$ Los gastos en la esfera social se enmarcarán en las posibilidades reales de recursos financieros que se generan en la economía del país.

$\diamond \quad$ Establecer relaciones más efectivas entre el consumo por ingresos provenientes del trabajo y los fondos sociales de consumo.

$\diamond \quad$ Adecuar la expansión de los servicios sociales al dinamismo de los sectores productivos de bienes y servicios.

$\diamond \quad$ Sistema tributario como elemento redistribuidor del ingreso.

$\diamond \quad$ Continuar preservando las conquistas en salud, educación, cultura, deporte, recreación, seguridad y asistencia social.

$\diamond \quad$ Reducir gastos excesivos.

$\diamond \quad$ Disminuir la participación relativa del presupuesto del Estado en el financiamiento de la seguridad social y seguir extendiendo la contribución de los trabajadores del sector estatal y la aplicación de regímenes especiales de contribución en el sector no estatal.

$\diamond \quad$ Eliminar gratuidades indebidas y subsidios personales excesivos, estableciendo compensaciones a las personas necesitadas.

$\diamond \quad$ Eliminar la libreta de abastecimiento.

$\diamond \quad$ Seguridad social y focalización hacia las vulnerabilidades: perfeccionar vías de protección a población vulnerable y garantizar que la asistencia social la reciban las personas que realmente la necesitan.

Puede observarse que un importante conjunto de lineamientos supone la modificación de las proporciones entre los grupos de la estructura de la propiedad sobre los medios de producción, de las fuentes de ingresos y del empleo, y de la importancia del rol de los distintos sectores de propiedad en la economía, con lo que se alterará sustantivamente la estructura actual de estratificación social. Uno 
de los aportes principales de las investigaciones precedentes es mostrar que los activos necesarios para aprovechar las nuevas oportunidades de empleo (conocimientos, capital, bienes para generar actividades mercantiles, información, conexiones que permitan acceder a los nuevos espacios económicos creados, etc.) no están distribuidos de forma equitativa entre todos los grupos sociales.

Sin embargo, la estrategia de cambio contenida en los citados lineamientos, no prevé, al menos por el momento, acciones afirmativas para atender estas desventajas y proveer de activos o de capacidades para incrementarlos, a los grupos carentes de ellos, a través de la capacitación, la asesoría jurídica, el apoyo financiero, la información, entre otras opciones posibles. Por ello es de esperar, entre los efectos principales de las medidas de cambio, la dinamización de corrientes de movilidad social ascendente $y$ descendente, al crear nuevas oportunidades que podrán ser aprovechadas en mayor o menor proporción en dependencia del conjunto de activos tangibles e intangibles de los que se disponga. situar:

Entre los efectos esperables se pueden

\section{1) Diversificación de las fuentes de ingreso y empleo}

Hasta hoy, aunque en mucha menor proporción que en los 80 , el sector estatal de la economía ha sido el mayor empleador del país. Se prevé que en un plazo relativamente breve, este sector se descargue de alrededor de un millón de trabajadores, que deberán ser absorbidos por el cooperativismo, el trabajo por cuenta propia, la actividad agrícola usufructuaria, nuevas empresas mixtas, entre otras.

\section{2) Ampliación de la franja de pobreza}

Al menos en un primer momento, es de esperar que grupos poblacionales que saldrán del empleo estatal o perderán la protección de la asistencia social y que tienen baja capacidad para generar sus propios ingresos, por carencia de activos $y$ hacer frente al incremento del peso del mercado en la satisfacción de necesidades básicas, experimenten un proceso de empobrecimiento.

\section{3) Ensanchamiento de brechas de equidad precedentes}

De género, raza, territoriales, generacionales, debido a la repetición de un patrón de movilidad selectivo basado en activos familiares $y$ personales.

\section{4) Marginalización social}

Investigaciones recientes mostraron la tendencia a la creación de asentamientos espontáneos, asociados a las carencias de vivienda, de empleo adecuado y a la migración desde territorios de menor grado de desarrollo hacia otros con mayores oportunidades económicas, donde las poblaciones viven en condiciones muy precarias $y$ formalmente fuera de los beneficios que ofrecen las coberturas universales de algunos servicios públicos esenciales y de los espacios de participación local-comunitaria establecidos, cuya distribución corre a cuenta de las estructuras de gobierno, sectoriales territoriales y de la residencia legal.

Estos procesos de marginalización espacial podrían debilitarse en la nueva etapa, si los avances esperados en las políticas de desarrollo local y en la construcción de viviendas frenan las migraciones internas; o podrían fortalecerse si la brecha territorial estimula los movimientos hacia centros de mayores oportunidades.

\section{5) ACTIVACIÓN DE MICROESCENARIOS, MICROPRÁCTICAS Y ESTRATEGIAS FAMILIARES DE SOBREVIVENCIA Y AMPLIACIÓN DE LOS INGRESOS}

De la estrategia planificada de reforma, concebida "desde arriba" (los lineamientos) se infiere un mayor protagonismo de los microescenarios, especialmente por los nuevos roles que se concede a los gobiernos municipales, a los proyectos de desarrollo local y a las empresas. Ello generará necesariamente una activación formal de los microespacios, que se verá reforzada por prácticas no formales ni 
planificadas centralmente, que son aquellas que se generan "desde abajo", como parte de la adaptación creativa desde el espacio familiar y comunitario a las circunstancias de cambio. En el período de reforma precedente (años 90), este resultó uno de los escenarios más cambiantes $y$ activos $y$ es previsible que suceda ahora lo mismo, junto a una ampliación de la franja de empleo informal y dentro de este, de sus ámbitos precarios.

\section{CONSIDERACIONES FINALES}

Observando en conjunto la experiencia de la política social cubana de los últimos 50 años, resaltan al menos como elementos de buenas prácticas:

$\diamond \quad$ Una consideración del ser humano y de sus necesidades básicas amplia e integral, esto es, que no las restringe a la alimentación y a los servicios básicos, sino que incluye en estas relevantes elementos de la espiritualidad y la cultura.

$\diamond \quad$ La primacía de la equidad sobre el eficientismo.

$\diamond \quad$ Universalización radical, expresada en servicios de cobertura total a partir de la demanda real.

$\diamond \quad$ Permanencia y estabilidad de las estrategias en el tiempo.

$\diamond \quad$ Responsabilidad del Estado.

$\diamond \quad$ Elevado peso de la inversión en elementos de desarrollo (salud y educación).

$\diamond \quad$ Amplio perfil de la asistencia social.

$\diamond \quad$ Integración a partir del empleo.

$\diamond \quad$ Colocación de las estrategias de alivio a la pobreza dentro de estrategias más amplias de desarrollo social, con menor peso del asistencialismo.

Entre las limitaciones que deberían quedar como lecciones aprendidas, los estudios sobre el tema señalan: débil sustentabilidad económica de la política social, débil retorno de la inversión social hacia la economía (reflejado en un bajo efecto de la elevación de la instrucción y la calificación sobre los niveles de productividad y la innovación tecnológica), baja articulación entre los resultados del trabajo y el acceso al bienestar, predominio de las estrategias sectoriales que obstaculizan la concepción integradora del desarrollo social, excesivo énfasis en el consumo social estatalmente normado en detrimento de la esfera familiar autónoma de elección de satisfactores de necesidades, absolutización del estatalismo y excesivo centralismo, baja posibilidad de participación en la toma de decisiones de los actores locales (gubernamentales y no gubernamentales), fallas de focalización y poco uso de políticas de acción afirmativa lo cual tiene como efecto la reproducción de desventajas de grupos históricamente preteridos, que no pueden aprovechar en paridad las condiciones favorables generales creadas (por ejemplo, negros, mujeres, ancianos, comunidades en territorios de mayor retraso relativo), priorización desbalanceada de dimensiones del desarrollo social lo que genera déficits acumulados en áreas relevantes asociadas a la situación familiar (fundamentalmente en lo relacionado con el acceso a una vivienda $y$ un hábitat familiar adecuados y empleos con ingresos suficientes) (Espina, 2008: 142).

Un examen de las directrices de la nueva reforma evidencia que esta posee potencialidades para superar las limitaciones señaladas a la política social, especialmente a través de la descentralización territorial y empresarial, el énfasis en el desarrollo local y la diversificación de fuentes de empleo e ingresos, todo en un contexto que conserva las políticas universales básicas. Es de esperar que ello permita una mejor expresión y atención a las diversidades. Pero también se pone de manifiesto que esta plataforma de cambio es portadora de potencialidades para el deterioro de la equidad, asociadas al ensanchamiento del rol del mercado en la distribución de bienes y servicios, en circunstancias en que una franja significativa de la población, concentrada en grupos sociales específicos, no alcanza estándares de ingresos y calidad de vida básicos. Al menos en el corto plazo, los impactos que pueden preverse, especialmente en el mundo del trabajo, serán muy contradictorios y ambivalentes.

El escenario que prevalezca dependerá de la presencia de políticas intencionadas de equidad social, las cuales no aparecen claramente 
expresadas en los documentos actuales, lo que coloca en un primer plano, el ámbito de la política social. Es necesario que la reforma reconsidere y ponga mayor énfasis en una política de equidad que tenga entre sus ejes centrales la dimensión empleo-ingresos. En esta dirección, algunas propuestas a analizar serían:

$\diamond \quad$ Instalar sistemas de información sobre posibilidades de empleo, asistencia social y servicios a nivel municipal, de amplia visibilidad $y$ fácil acceso.

$\diamond \quad$ Asumir la concepción del territorio como factor de desarrollo. En esta concepción juega un papel esencial la identificación de los actores socioeconómicos locales, en tanto agentes del cambio, como requisito metodológico indispensable en el diseño de programas de desarrollo o acciones autotransformativas a escala local. Se trataría de activar los territorios, no solo en el sentido económico previsto en los lineamientos, sino también como escenario de políticas sociales y en la potenciación de la innovación y la instalación de una capacidad perdurable de autogestión y autoorganización participativa de las sociedades locales.

$\diamond \quad$ Sistematizar en el diseño de políticas la realización de diagnósticos previos basados en el estado de la equidad a nivel nacional, provincial, municipal y comunitario; asimismo, la inclusión de acciones directas de afirmación para los grupos en desventaja.

$\diamond \quad$ Ampliación de los contenidos y límites de la participación en la construcción de la agenda social, priorizando sus elementos de cogestión, formulación estratégica y control popular del proceso $y$ sus resultados.

$\diamond \quad$ Producir cambios en el sistema de seguridad social. Además de los previstos en los lineamientos, establecer un fondo opcional de pensiones contributivas $y$ ampliar el tiempo de amparo y las compensaciones o apoyos para emprendimientos productivos para los trabajadores que queden fuera del empleo estatal.
Otorgar un tratamiento especial a la mujer madre jefa de hogar en esta situación.

$\diamond \quad$ Sistematizar la evaluación periódica de los resultados de las políticas sociales, incorporando entre los indicadores básicos sus impactos sobre la equidad social, la situación del empleo, particularmente de la mujer y de los grupos en desventaja en general.

$\diamond \quad$ Otorgar créditos preferenciales a grupos en desventaja para el desarrollo de emprendimientos productivos, individuales, familiares y en cooperativas.

$\diamond \quad$ Promover el establecimiento de sistemas laborales más flexibles (trabajo a media jornada o solo algunos días a la semana, horario abierto, trabajo a domicilio) para las mujeres jóvenes con hijos pequeños y a cargo de personas dependientes como discapacitados $y$ adultos mayores. Hacer extensivo este régimen a hombres que asuman el rol de cuidadores.

$\diamond \quad$ Ampliación de los servicios para la tercera edad. Generar políticas de proximidad con instituciones multiuso que atiendan necesidades de cuidado, médicas, de tiempo libre, etc. de los ancianos en su entorno. Estos servicios pueden ser coordinados por el Estado, pero suministrados por actores diversos.

\section{BIBLIOGRAFÍA}

\section{LIBROS Y CAPÍTULOS DE LIBROS}

Álvarez, Mayda. "Mujer y poder en Cuba". Cuba construyendo futuro. Monereo, M. et ál. (coord.). España: El Viejo Topo, 2000.

Echevarría, Dayma. "Mujer, empleo y dirección en Cuba: algo más que estadísticas". 15 años del Centro de Estudios de la Economía Cubana. Cuba: Editorial Félix Varela, 2004.

Espina, Mayra. Políticas de atención a la pobreza y la desigualdad. Examinando el rol del Estado en la experiencia cubana. Argentina: Colección CLACSO-CROP, 2008.

Ferriol, Ángela. "Política social y desarrollo. Una aproximación global”. Política 
social y reformas estructurales: Cuba a principios del siglo XXI. Álvarez, E. y Mattar, J. (coords.). México: CEPAL-INIEPNUD, 2004.

Oficina Nacional de Estadísticas (Cuba). Censo de Población y Viviendas. Cuba: Ministerio de Economía y Planificación, 2006.

Oficina Nacional de Estadísticas (Cuba). Anuario Estadístico de Cuba 2009. Cuba: Ministerio de Economía y Planificación, 2010.

Partido Comunista de Cuba. Lineamientos económicos y de la política social. Cuba: Editora Política, 2011.

Pérez, Victoria. "Impacto del período especial en la vida cotidiana de la mujer cubana en la década de los años 90". Crisis, cambios económicos y subjetividad de las cubanas. Colectivo de autores. Cuba: Editorial Félix Varela, 2004.

ARTÍCULOS EN REVISTAS

Espina, Mayra. "Viejas y nuevas desigualdades en Cuba. Ambivalencias y perspectivas de la reestratificación social". Nueva Sociedad 216. Buenos Aires. Julio-agosto 2008: 133-149.

\section{TEXTOS INÉDITOS}

Departamento de Estudios sobre Familia. "Familia y cambios socioeconómicos a las puertas del nuevo milenio". Informe de Investigación. La Habana: Centro de Investigaciones Psicológicas y Sociológicas, 2001.

García, Anicia y Anaya, Betsy. "Política social en Cuba, nuevo enfoque y programas recientes". Ponencia presentada en el Seminario del Centro de Estudios de la Economía Cubana. La Habana, 2006.

Nerey, Boris. "El modelo de desarrollo y estado de bienestar en Cuba". [Tesis de Maestría en Sociología]. Universidad de La Habana, 2000.

Fecha de ingreso: 29/11/2011 Fecha de aprobación: 10/02/2012 